\title{
Bullous Pemphigoid Triggered by Liraglutide
}

\author{
Mary-Katharine Collins, MD; Sonal Choudhary, MD; Jonhan Ho, MD; Yuri L. Bunimovich, MD, PhD
}

\section{PRACTICE POINTS}

- Liraglutide and dipeptidyl peptidase 4 inhibitors, medications used in the treatment of diabetes mellitus, may be linked to the development of bullous pemphigoid (BP).

- Further study of the mechanism of action of these medications may lead to improved understanding of the pathogenesis of BP.

To the Editor:

Bullous pemphigoid (BP) is an autoimmune blistering disease that typically affects the elderly, with an incidence of approximately 7 new cases per million. ${ }^{1}$ The pathogenesis of BP involves autoantibodies to BP antigens 180 and 230 at the dermoepidermal junction. Bullous pemphigoid has been associated with the use of multiple medications; vaccines; and physical damage to the skin, including trauma, radiation, and surgery. ${ }^{2}$

Several classes of medications may cause BP; one study described an association of BP with loop diuretics, ${ }^{3}$ while others found higher incidences of BP in patients taking aldosterone antagonists and neuroleptics. ${ }^{4}$ We describe a case of drug-triggered BP to liraglutide, a glucagonlike peptide 1 (GLP-1) receptor agonist.

A 75-year-old man presented to dermatology for evaluation of a vesicular eruption on the head, neck, trunk, and arms of 6 months' duration. The eruption developed 2 weeks after starting liraglutide $1.2 \mathrm{mg}$ subcutaneously daily for diabetes mellitus. The patient had a medical history of type 2 diabetes mellitus, hypertension, stroke, and prostate cancer treated with prostatectomy, and he also was taking insulin. Liraglutide was discontinued shortly after the onset of the eruption.

Physical examination revealed annular plaques on the head, neck, trunk, and arms with central hypopigmentation and hyperpigmented borders (Figure 1). Two tense bullae were evident on the left flank (Figure 2). Histopathology revealed a subepidermal blister, mixed perivascular infiltrate with numerous eosinophils, and pigment incontinence (Figure 3). Direct immunofluorescence showed linear deposition of IgG and C3 along the basement membrane zone that was localized to the roof of the blister on salt-split analysis. No microorganisms were identified on periodic acid-Schiff, GrocottGomori methenamine-silver, acid-fast bacilli, and Fite stains. The patient initially was treated with clobetasol ointment $0.05 \%$, leading to marginal improvement. He declined treatment with prednisone or dapsone, and he was started on doxycycline. Seven months after stopping liraglutide and starting doxycycline, the patient had no blisters, but residual pigmentary changes remained.

Two types of BP have been described in response to medications: drug-induced $\mathrm{BP}$ and drug-triggered BP. Drug-induced BP presents as an acute, self-limited eruption that typically resolves after withdrawal of the offending agent. It tends to involve a younger population and may present with mucosal involvement and target lesions on the palms and soles. Direct immunofluorescence shows linear IgG and C3 deposition at the basement membrane zone. Patients tend to respond quickly to systemic corticosteroids and have low recurrence rates. Drug-triggered BP is a chronic form of BP that is caused by a medication and is not resolved with removal of the offending agent. ${ }^{5}$ Therefore, drug-triggered BP is

From the Department of Dermatology, University of Pittsburgh, Pennsylvania. Dr. Choudhary also is from the Department of Dermatopathology, and Dr. Ho also is from the Department of Pathology.

The authors report no conflict of interest.

Correspondence: Yuri L. Bunimovich, MD, PhD, Shadyside Place, 580 S Aiken Ave, Ste 201, Pittsburgh, PA 15232 (bunimovichyl@upmc.edu). doi:10.12788/cutis.0262 


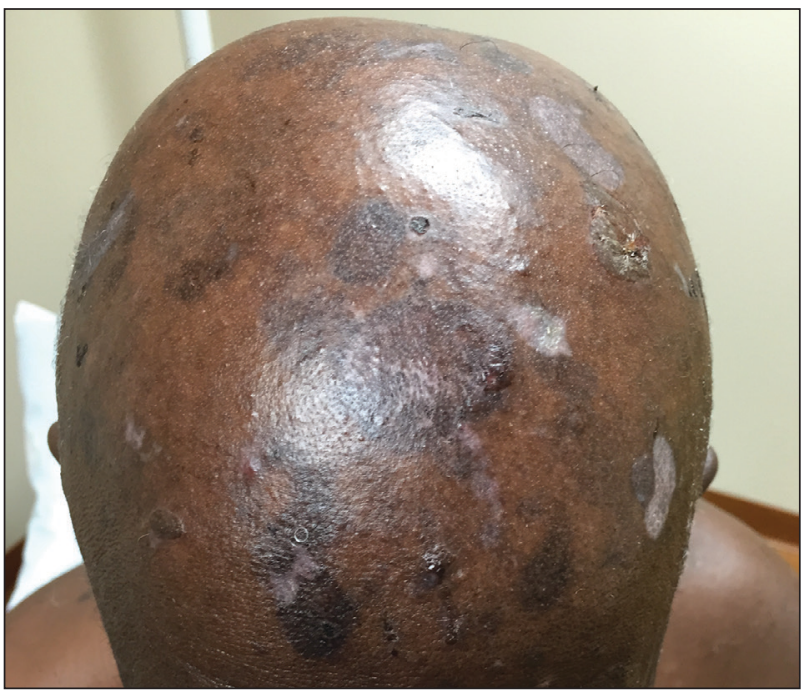

FIGURE 1. Drug-triggered bullous pemphigoid. Hypopigmented atrophic plaques and hyperpigmented plaques on the scalp.

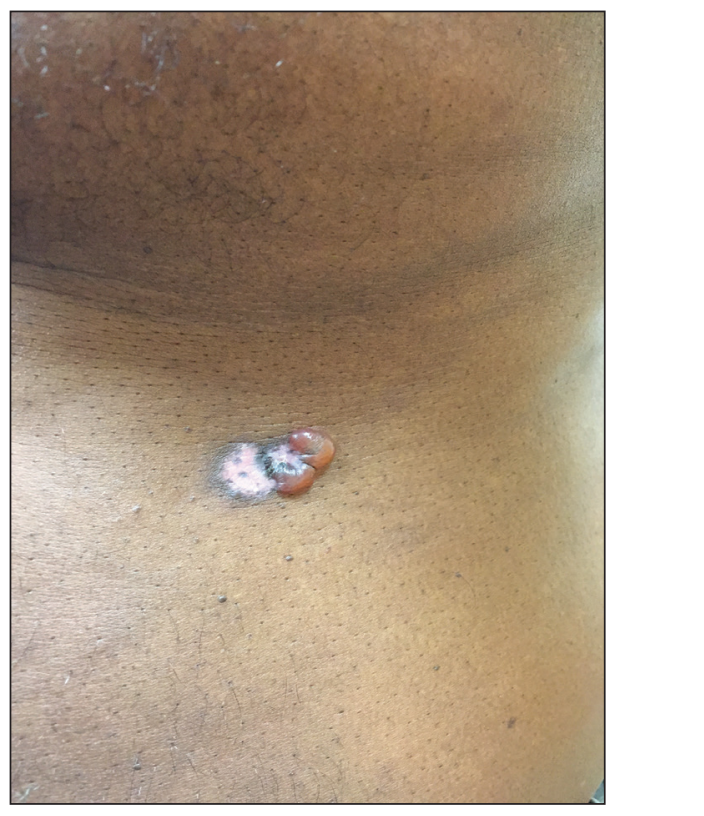

FIGURE 2. Drug-triggered bullous pemphigoid. Two vesicles at the periphery of a hypopigmented atrophic plaque on the left flank.

more difficult to detect, especially in patients taking multiple medications.

Our patient represents a case of drug-triggered BP to liraglutide. Liraglutide is a GLP-1 receptor agonist that is US Food and Drug Administration approved for the treatment of type 2 diabetes mellitus. Glucagonlike peptide 1 is an incretin hormone that is secreted by the intestine during digestion. It binds to the GLP-1 receptor leading to an increase in glucose-dependent insulin secretion and a decrease in glucagon secretion. ${ }^{6}$ Glucagonlike peptide 1 agonists also affect the
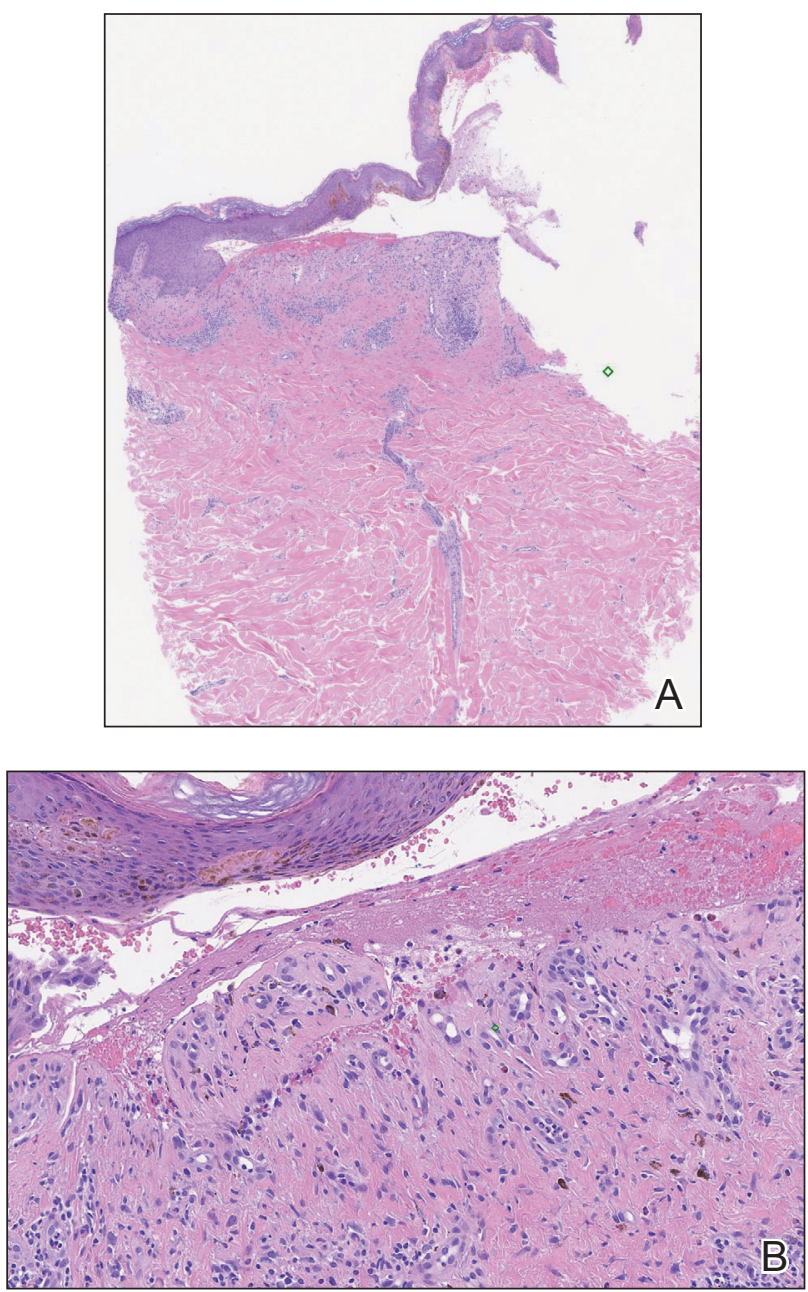

FIGURE 3. A, Histopathology showed a subepidermal blister $(H \& E$, original magnification $\times 4)$. B, Perivascular dermatitis with eosinophils and pigment incontinence also was present (H\&E, original magnification $\times 40$ ).

immune system; liraglutide has been shown to modestly improve psoriasis, reduce the number of dermal gamma delta T cells, and decrease IL-17 expression. ${ }^{7}$ Glucagonlike peptide 1 agonists also produce anti-inflammatory effects on multiple organs including the liver, brain, vasculature, kidney, and skin. ${ }^{8}$

Dipeptidyl peptidase 4 (DPP-4) inhibitors that function to inhibit the degradation of GLP-1 and other peptides also have been reported to cause BP. In several patients, the DPP-4 inhibitors vildagliptin and sitagliptin caused drug-induced BP that resolved with discontinuation of the medication. ${ }^{9}$ Dipeptidyl peptidase 4 is expressed in various organ systems including the skin, and inhibition of DPP-4 enhances eosinophil mobilization in the blood and recruitment to the skin in animal models. ${ }^{10}$

Although the pathogenesis of BP involves autoantibodies to BP antigens 180 and 230, these antibodies are not sufficient to cause disease, as antibasement antibodies 
have been detected in patients without clinically evident BP. These patients, however, may be more susceptible to developing medication-induced BP. Several hypotheses regarding the pathogenesis of medication-induced $\mathrm{BP}$ have been proposed, including immune dysregulation, molecular mimicry, and cross-reactivity to a prior sensitizing agent. ${ }^{5}$ Liraglutide and the DPP-4 inhibitors affect the immune system, supporting the hypothesis of immune dysregulation; however, the exact mechanism of how immune modulating medications such as GLP-1 agonists and DPP-4 inhibitors cause BP remains unclear.

The effects of liraglutide and the DPP-4 inhibitors on the immune system may play a role in the pathogenesis of drug-triggered BP and drug-induced BP, respectively. Additional studies of the immunomodulatory effects of GLP-1 agonists and DPP-4 inhibitors may help elucidate the pathogenesis of drug-triggered or drug-induced BP.

\section{REFERENCES}

1. Serwin AB, Musialkowska E, Piascik M. Incidence and mortality of bullous pemphigoid in north-east Poland (Podlaskie Province), 1999-2012: a retrospective bicentric cohort study. Int J Dermatol. 2014;53:E432-E437.
2. Danescu S, Chiorean R, Macovei V, et al. Role of physical factors in the pathogenesis of bullous pemphigoid: case report series and a comprehensive review of the published work. J Dermatol. 2016;43:134-130.

3. Lloyd-Lavery A, Chi CC, Wojnarowska F, et al. The associations between bullous pemphigoid and drug use: a UK case-control study. JAMA Dermatol. 2013;149:58-62.

4. Bastuji-Garin S, Joly P, Picard-Dahan C, et al. Drugs associated with bullous pemphigoid. a case-control study. Arch Dermatol. 1996;132:272-276.

5. Stavropoulos PG, Soura E, Antoniou C. Drug-induced pemphigoid: a review of the literature. J Eur Acad Dermatol Venereol. 2014;28:1133-1140.

6. Triplitt C, Solis-Herrera C. GLP-1 receptor agonists: practical considerations for clinical practice. Diabetes Educ. 2015;41(suppl 1):32S-46S.

7. Buysschaert $M$, Baeck $M$, Preumont $V$, et al. Improvement of psoriasis during glucagon-like peptide-1 analogue therapy in type 2 diabetes is associated with decreasing dermal gammadelta T-cell number: a prospective case-series study. Br J Dermatol. 2014;171:155-161.

8. Lee YS, Jun HS. Anti-inflammatory effects of GLP-1-based therapies beyond glucose control. Mediators Inflamm. 2016;2016:3094642.

9. Skandalis K, Spirova M, Gaitanis G, et al Drug-induced bullous pemphigoid in diabetes mellitus patients receiving dipeptidyl peptidase-IV inhibitors plus metformin. J Eur Acad Dermatol Venereol. 2012;26:249-253.

10. Forssmann U, Stoetzer C, Stephan M, et al. Inhibition of CD26 /dipeptidyl peptidase IV enhances CCL11/eotaxin-mediated recruitment of eosinophils in vivo. J Immunol. 2008;181:1120-1127. 